\title{
Hypoxia Induces Osteogenesis-Related Activities and Expression of Core Binding Factor $\alpha 1$ in Mesenchymal Stem Cells
}

\author{
Jiao Huang, ${ }^{1}$ Feng Deng, ${ }^{2}$ Lu Wang, ${ }^{3}$ Xue-Rong Xiang, ${ }^{1}$ Wen-Wen Zhou, ${ }^{3} \mathrm{Na} \mathrm{Hu}{ }^{3}$ \\ and Ling $\mathrm{Xu}^{3}$ \\ ${ }^{1}$ Department of Periodontology, Affiliated Hospital of Stomatology, Chongqing University of Medical Sciences, \\ Chongqing, P.R. China \\ ${ }^{2}$ Department of Orthodontics, Affiliated Hospital of Stomatology, Chongqing University of Medical Sciences, \\ Chongqing, P.R. China \\ ${ }^{3}$ Department of Prosthodontics, Affiliated Hospital of Stomatology, Chongqing University of Medical Sciences, \\ Chongqing, P.R. China
}

\begin{abstract}
Mesenchymal stem sells (MSCs) have received much attention in the field of bone tissue engineering due to their biological capability to differentiate into osteogenic lineage cells. Hypoxia-inducible factor 1alpha (HIF-1 $\alpha$ ) plays an important role in the MSC-related bone regeneration during hypoxia, while core binding factor alpha $1(\mathrm{Cbf} \alpha 1)$ is a transcription regulator that is involved in the chondrocyte differentiation and ossification. In the present study, we investigated the effects of hypoxia on biological capability of MSCs. MSCs were isolated from adult rabbit bone marrow, and were cultured in vitro under normoxia (air with $5 \%$ $\mathrm{CO}_{2}$ ) or hypoxia $\left(5 \% \mathrm{CO}_{2}\right.$ and $\left.95 \% \mathrm{~N}_{2}\right)$. The proliferation of MSCs, alkaline phosphatase (ALP) activity, and production of collagens type I and type III (Col I/III) were examined. The expression levels of HIF- $1 \alpha$ and $\mathrm{Cbf} \alpha 1$ were measured by real-time PCR and western blot analyses. We found that hypoxia significantly induced the proliferation of MSCs and increased ALP activity and the production of Col I/III. Moreover, hypoxia increased the expression of $\operatorname{Cbf} \alpha 1$ mRNA after $12 \mathrm{~h}$, whereas the expression of HIF-1 $\alpha$ mRNA was increased after $1 \mathrm{~h}$ of hypoxia. Knockdown of HIF-1 $\alpha$ expression with a small interfering RNA significantly increased the expression levels of $\mathrm{Cbf} \alpha 1$ protein either under the normoxia or hypoxia condition. Our results indicate that hypoxia enhances MSCs to differentiate into osteogenic lineage cells and suggest that $\mathrm{Cbf} \alpha 1$ may be negatively regulated by $\mathrm{HIF}-1 \alpha$.
\end{abstract}

Keywords: mesenchymal stem cells; hypoxia; differentiation; HIF-1 $\alpha$; Cbf $\alpha 1$

Tohoku J. Exp. Med., 2011, 224 (1), 7-12. 2011 Tohoku University Medical Press

Mesenchymal stem sells (MSCs) have received extensive attention in the field of bone tissue engineering due to their distinct biological capability to differentiate into osteogenic lineage cells (Otto and Rao 2004; GraneroMolto et al. 2008). Physiological microenvironments, such as oxygen concentration and hypoxia, play a crucial role in the process of MSC-related bone regeneration and development (Mohyeldin et al. 2010). Hypoxia-inducible factor 1alpha (HIF-1 $\alpha$ ), the hallmark of hypoxia response factor, plays an important role in the differentiation of MSCs into osteogenic lineages (Nagano et al. 2010; Liu et al. 2011). The expression of HIF- $1 \alpha$ was decreased with the time of hypoxia, whereas the expression of core binding factor alpha $1(\mathrm{Cbf} \alpha 1)$, another key transcription regulator involved in the chondrocyte differentiation and ossification, was increased gradually (Rabie et al. 2004; Augello and De Bari 2010). However, other report showed that temporary exposure to hypoxia would inhibit the expression of
Cbf $\alpha-1 /$ Runx2 in MSCs (Potier et al. 2007).

Differentiation of MSCs into osteogenic lineages and the osteogenesis-related change of MSCs are the complicated processes, and are regulated by many growth factors, hormones, protein kinases and inhibitors. The regulation of growth factors and hormones, such as transform growth factor beta (TGF- $\beta$ ), fibroblast growth factor (FGF), insulin-like growth factor 1 (IGF-1), bone morphogenetic protein (BMP) and parathormone (PTH), on MSCs proliferation and differentiation have been studied thoroughly (Haddad and Harb 2005; Qi et al. 2009; Huang et al. 2010). It has been reported that MSCs cultured in vitro would differentiate into osteoblasts spontaneously (Seong et al. 2010; Mikami et al. 2011). But whether HIF- $1 \alpha$ and $\mathrm{Cbf} \alpha 1$ are involved in the proliferation and differentiation of MSCs as well as the osteogenesis-like changes under hypoxia has not been revealed, and also little is known about the regulation of $\mathrm{Cbf} \alpha 1$ during the differentiation of MSCs into osteogenic

Received January 31, 2011; revision accepted for publication April 4, 2011. doi: 10.1620/tjem.224.7

Correspondence: Dr. Ling Xu, Department of Prosthodontics, Affiliated Hospital of Stomatology, Chongqing University of Medical Sciences, Chongqing 400015, P.R. China.

e-mail: xulingcqxy@163.com 
lineage cells. In the present study, we examined the effects of hypoxia on expression levels of HIF- $1 \alpha$ and $\mathrm{Cbf} \alpha 1$ in MSCs.

\begin{abstract}
Methods
Propagation of rabbit bone marrow MSCs

MSCs were isolated from male rabbit marrow and characterized according to a previous report (Lee et al. 2004). Briefly, rabbit marrow $(1 \mathrm{ml})$ was collected from the anterior superior iliac spine of healthy New Zealand white rabbits (body weight $2.4 \pm 0.3 \mathrm{~kg}$ ). The study was performed with the approval by the institutional review board of the Chongqing Veterans General Hospital. Mononuclear cells were obtained by negative immunodepletion of CD34, CD44 and CD105 using a commercially available kit (RosetteSep, StemCell Technologies, Vancouver, Canada) according to the manufacturer's instructions, followed by Ficoll-Paque density-gradient centrifugation (1.077 $\mathrm{g} / \mathrm{cm}^{3}$ ), and plated in tissue culture plates in expansion medium. The expansion medium consisted of Iscove's modified Dulbecco's medium (IMDM) (Gibco Inc., USA) and 10\% fetal bovine serum (Hyclone Inc., Logan, Utah, USA) supplemented with $10 \mathrm{ng} / \mathrm{mL}$ epidermal growth factor (EGF) (Becton Dickinson Inc., Franklin Lakes, New Jersey, USA) and $10 \mathrm{ng} / \mathrm{mL}$ basic fibroblast growth factor (bFGF) (R\&D Systems Inc., Minneapolis, MN, USA). The adherent cells were amplified within the expansion medium.
\end{abstract}

\section{Establishment of hypoxic culture model}

To establish a hypoxic model of culturing MSCs in vitro, MSCs were washed twice with medium under pure nitrogen pre-saturated and incubated in a special chamber containing $5 \% \mathrm{CO}_{2}$ and $95 \% \mathrm{~N}_{2}$. Cells were divided into two groups: (i) Normoxia group, cells incubated at $37^{\circ} \mathrm{C}$ in humid air with $5 \%(\mathrm{v} / \mathrm{v}) \mathrm{CO}_{2}$; (ii) Hypoxia group, cells incubated in a special chamber containing $5 \% \mathrm{CO}_{2}$ and $95 \% \mathrm{~N}_{2}$.

\section{Measurement of cellular proliferation}

In order to assess the effects of hypoxia on MSCs proliferation, we measured DNA synthesis of MSCs by quantifying the radioactivity of ${ }^{3} \mathrm{H}$-thymidine $\left({ }^{3} \mathrm{H}-\mathrm{TdR}\right)$ incorporated into cells. Cells were cultured in 6-well plates for $16 \mathrm{hrs} .37 \mathrm{kBq}$ of ${ }^{3} \mathrm{H}$-TdR (NEN Life Science, Boston, MA, USA) was added to each well 4 hours before harvested. MSCs were washed twice with $100 \mathrm{ml} / \mathrm{L}$ trichloroacetic acid, centrifuged and resuspended, and continuously incubated for 30 minutes at $60^{\circ} \mathrm{C}$ with $0.5 \mathrm{ml} \mathrm{NaOH}(0.3 \mathrm{~mol} / \mathrm{L})$. Finally, the cell lysates were collected, and the radioactivity was measured in a liquid scintillation counter (Beckman Inc., CA, USA). The experiment was independently performed three times.

\section{Alkaline phosphatase (ALP) assay}

Cellular proteins were solubilized with $1 \%$ Triton $X-100$ in $0.9 \% \mathrm{NaCl}$ and centrifuged. Supernatants were assayed for ALP activity with alkaline phosphatase yellow kit (Sigma Inc., St. Louis, MO, USA) according to the instructions of manufacturer. Protein concentration was calculated using the BCA protein assay kit (Pierce Biotechnology, Inc., IL, USA), and ALP activity was normalized to cellular protein content.

\section{Measurement of Collagens I and III by ELISA}

The cellular lysate for determination of collagen type I and type III (Col I/III) levels was collected from the normoxia group and hypoxia group, respectively. An ELISA was used to quantify cellular
Col I/III levels according to the manufacture instructions (Ximei Bio., Inc., Shanghai, China).

Quantitative estimation of HIF-1 $\alpha$ and Cbf $\alpha 1 \mathrm{mRNAs}$ by real-time RT-PCR

The total RNA of MSCs in each group was extracted using a total RNA isolation system kit (Promega Corp., Madison, WI, USA) for the detection of HIF- $1 \alpha$ and $\mathrm{Cbf} \alpha 1 \mathrm{mRNA}$ expression by SYBR green real-time PCR. cDNA was synthesized with the Reverse Transcription System kit (Promega Corp.). All primers were synthesized by Invitrogen Life Technologies (Invitrogen Inc., Carlsbad, CA, USA). cDNA $(1 \mu \mathrm{l})$ was mixed with $2 \times$ SYBR green PCR master mix (Applied Biosystems Corp., Carlsbad, CA, USA) and $300 \mathrm{nM}$ gene-specific primers in a final volume of $20 \mu \mathrm{l}$, and real-time PCR was performed (ABI PRISM 7000, PE Applied Biosystems Corp.). The values were normalized with $\beta$-actin mRNA as an internal standard.

\section{RNA interference for HIF-1 $\alpha$ and western blot analysis}

Cells were cultured in $60-\mathrm{mm}$ plates overnight in complete growth medium. Cells were transfected with small interfering RNA (siRNAs) $(10 \mathrm{nM})$ in Opti-MEM Medium (Invitrogen) using the CodeBreaker siRNA Transfection Reagent (Promega Corp.) according to the manufacture's instructions. The sequences of the siRNAs synthesized by Genechem Co. (Shanghai, China) were as follows: HIF-1 $\alpha$, CCG GAG AGG TGG ATA TGT CTG GGC TCG; scramble SiRNA (as a control), CCG GGG GTC TGT ATA GGT GGA GAC TCG. After cultured overnight, cells were subjected to hypoxia for $12 \mathrm{~h}$. Cells in each group were collected and Western blot assay was performed to detect $\mathrm{Cbf} \alpha 1$ expression as previously described (Zhao et al. 2010). The relative protein expression was calculated by the ratio of HIF- $1 \alpha$ and $\mathrm{Cbf} \alpha 1$ expression to the $\beta$-actin expression, respectively.

\section{Statistical analysis}

Data are expressed as the mean \pm S.E.M. The statistical significance about the difference between the means of the groups was determined by two-tailed Student's $t$ tests. $p<0.05$ was considered significant.

\section{Result}

\section{Hypoxia induces MSCs proliferation}

Proliferation of MSCs was assessed by ${ }^{3} \mathrm{H}-\mathrm{TdR}$ incorporation assay. The ${ }^{3} \mathrm{H}-\mathrm{TdR}$ incorporation value in the hypoxia group was significantly higher than that in the normoxia group, $p<0.05$ compared with hypoxia $(n=3$, Fig. $1)$.

\section{Hypoxia enhances the osteogenesis-related functions of} MSCs

ALP activity and production of Col I/III are the indicators of osteogenesis-related activities. ALP activity was markedly increased upon the treatment of hypoxia, $p<0.05$ compared with normoxia (Fig. 2A). The levels of Col I/III were measured by ELISA, showing that both of them were significantly increased in the hypoxia group, Col I, $p<0.05$ and Col III, $p<0.01$, compared with normoxia, respectively (Fig. 2B). These data suggest that hypoxia can promote the 
osteogenesis-like process of MSCs.

Effects of hypoxia on expression of HIF-1 $\alpha$ and Cbfal $m R N A s$ in MSCs

The expression levels of HIF- $1 \alpha$ and Cbf $\alpha 1$ mRNAs are measured by real-time PCR at different time point (Fig.

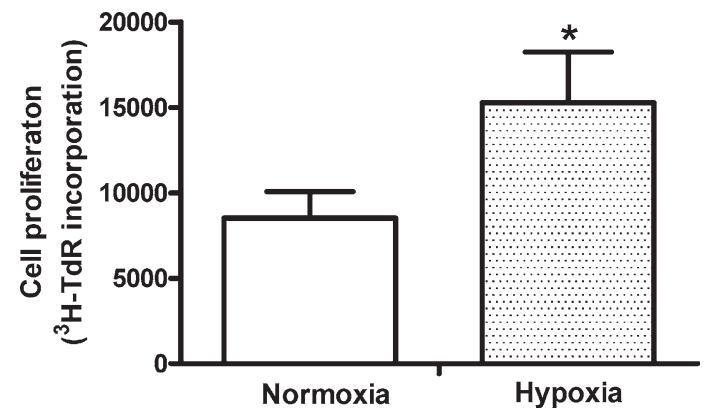

Fig. 1. Hypoxia induces MSC proliferation. Cells $\left(5 \times 10^{5}\right)$ are seeded in all 6 well plates and cultured for 16 hours. Proliferation is assessed by ${ }^{3} \mathrm{H}-\mathrm{TdR}$ incorporation assay during the final $4 \mathrm{~h}$ of the culture. The data is expressed as mean \pm S.E.M. of three independent experiments. $p<0.05$, normoxia versus hypoxia.
$3 \mathrm{~A}$ and $\mathrm{B})$. The HIF- $1 \alpha$ expression was significantly increased under hypoxia and gradually decreased with the time, $p<0.001$ at the time of $1 \mathrm{~h}, 6 \mathrm{~h}, 12 \mathrm{~h}$, and $p<0.05$ at the time of $24 \mathrm{~h}$, compared with the normoxia. In contrast, the $\mathrm{Cbf} \alpha 1$ expression was gradually increased with the time and significantly higher than that of normoxia at the time of $12 \mathrm{~h}$ and $24 \mathrm{~h}$ ( $p<0.01$ and $p<0.001$, respectively). We therefore speculated that the expression of HIF-1 $\alpha$ might be inversely related to the expression of $\mathrm{Cbf} \alpha 1$.

\section{Down-regulation of HIF-1 $\alpha$ induces Cbfal expression}

To explore the role of HIF-1 $\alpha$ in the regulation of Cbf $\alpha 1$ expression, HIF- $1 \alpha$ expression in MSCs was knockdowned with siRNA (Fig. 4A). The decrease in HIF-1 $\alpha$ expression obviously increased the expression levels of Cbf $\alpha 1$ protein either under the normoxia or hypoxia condition. Moreover, hypoxia significantly increased the $\mathrm{Cbf} \alpha 1$ protein expression (Fig. 4B), which is consistent with the result of mRNA expression (Fig. 3B). These data suggest that HIF-1 $\alpha$ may downregulate the $\mathrm{Cbf} \alpha 1$ expression and hypoxia.

A

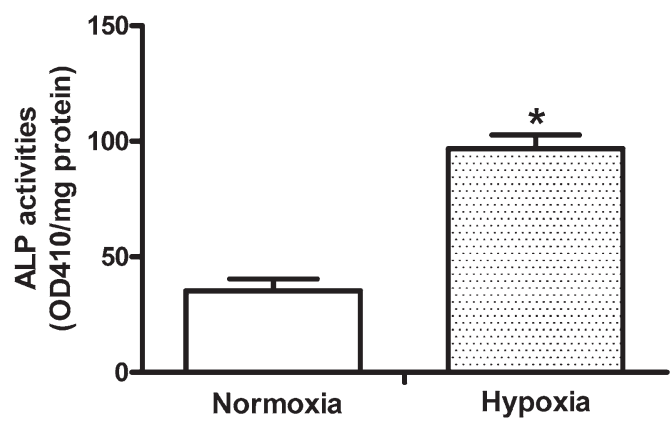

B
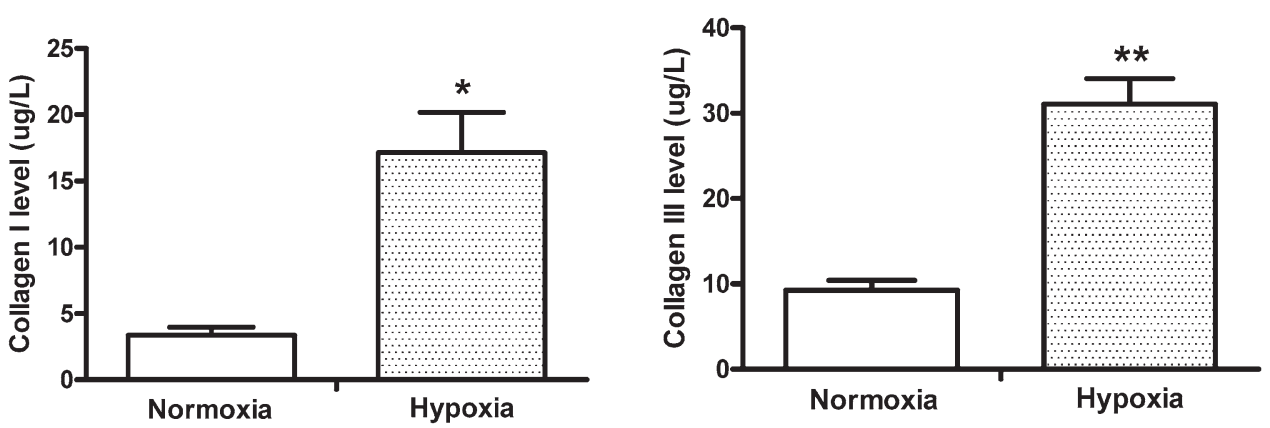

Fig. 2. Hypoxia induces osteogenesis of MSCs.

A) Quantitative assay of alkaline phosphatase activity. MSCs are cultured in 60-mm plates for $12 \mathrm{~h}$ and harvested for ALP activities measurement. ALP activity is normalized to cellular protein content. The data is expressed as mean \pm S.E.M. of three independent experiments. $p<0.05$, normoxia versus hypoxia. B) The content of collagen I and III is measured by ELISA assay. MSCs are cultured in 24-well plates and treated according to the experiment procedure for $24 \mathrm{hrs}$. Cellular lysate of each group are collected for determination of collagen I and III content, respectively. The data is expressed as mean \pm S.E.M. ${ }^{*} p<0.05, * * p<0.01$, normoxia versus hypoxia, $n=3$. 
A

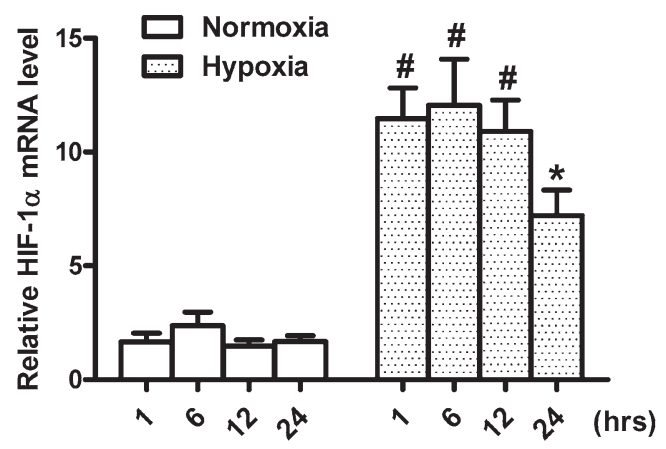

B

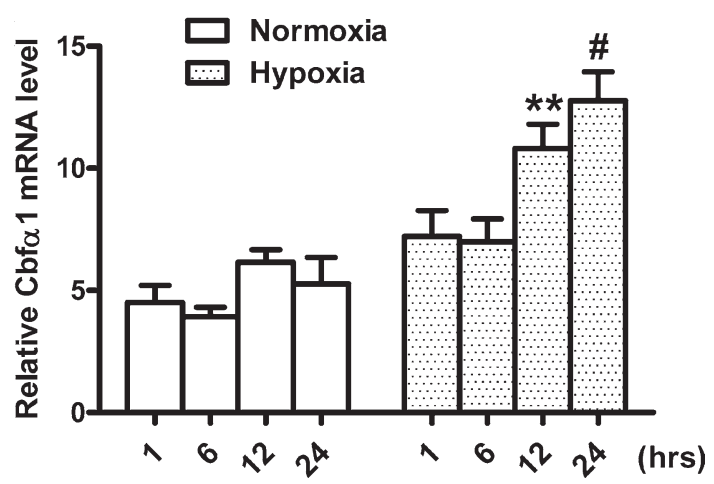

Fig. 3. Effects of hypoxia on HIF- $1 \alpha$ and $\mathrm{Cbf} \alpha 1$ expression in MSCs.

MSCs are treated according to the experiment procedure for $1 \mathrm{~h}, 6 \mathrm{~h}, 12 \mathrm{~h}$ and $24 \mathrm{~h}$. At different time-points, the total cellular RNA is extracted for detection of HIF- $1 \alpha$ and $\mathrm{Cbf} \alpha 1 \mathrm{mRNA}$ expression by SYBR green real-time PCR, respectively. The data is expressed as mean \pm S.E.M. $(n=3),{ }^{*} p<0.05, * * p<0.01, \# p<0.001$ versus normoxia, respectively.

\section{Discussion}

In the present study, we have shown that hypoxia markedly increases the proliferation of MSCs, ALP activity, an index of osteoblastic differentiation, and production of $\mathrm{Col}$ I/III. HIF- $1 \alpha$ is significantly increased at the early stage of hypoxia exposure, and later its level was decreased with exposure time. In contrast, the expression of $\mathrm{Cbf} \alpha 1$, a key transcription regulator of chondrocyte differentiation, is gradually increased with the exposure time of hypoxia. Further, down-regulation of HIF- $1 \alpha$ expression with SiRNA obviously induced $\mathrm{Cbf} \alpha 1$ expression either under normoxia or hypoxia. We therefore suggest that hypoxia may promote MSCs to differentiate into osteogenic lineage cells and that HIF- $1 \alpha$ may negatively regulate the $\mathrm{Cbf} \alpha 1$ expression.

Physiological microenvironments play a crucial role in maintenance of stem cell properties, and the oxygen con-
A

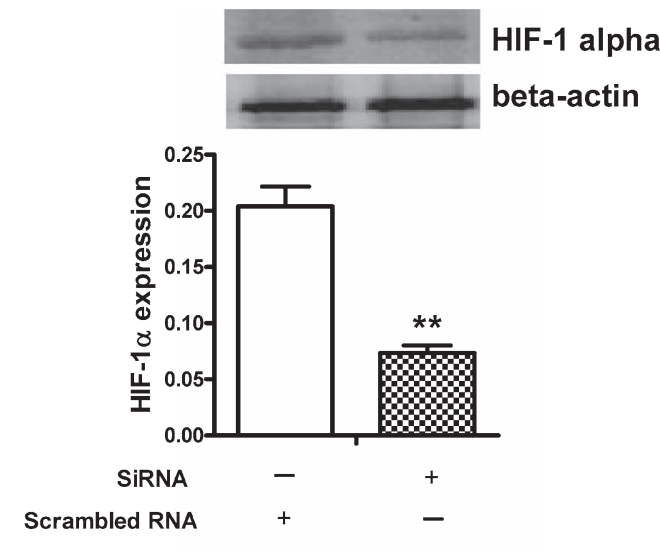

B
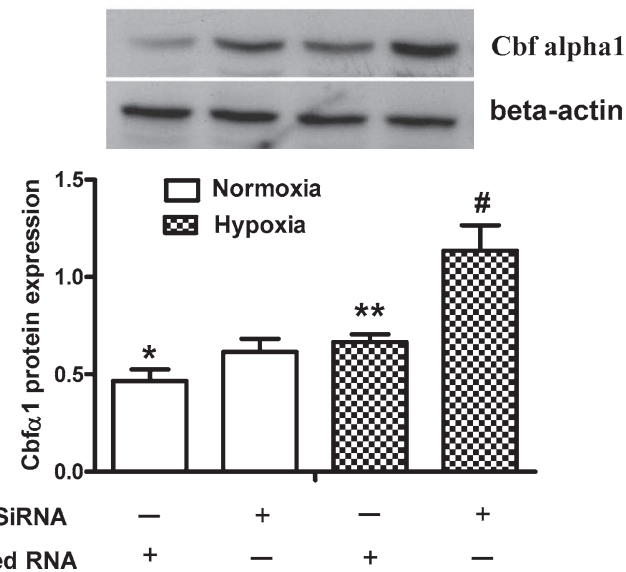

Fig. 4. Knockdown HIF- $1 \alpha$ expression induces $\mathrm{Cbf} \alpha 1$ expression.

A) MSCs are transfected with SiRNA of HIF-1 $\alpha$ and scrambled SiRNA respectively. $24 \mathrm{~h}$ later, the expression of HIF- $1 \alpha$ is detected by Western blot with specific antibody against HIF-1 $\alpha$. The filters are re-probed with $\beta$-actin antibody. B) $12 \mathrm{~h}$ after transfected with siRNA of HIF- $1 \alpha$, MSCs are subjected to normoxia and hypoxia for $12 \mathrm{~h}$, respectively. The protein level of $\mathrm{Cbf} \alpha 1$ is measured by Western blot with anti-Cbf $\alpha 1$ antibody. The filters are re-probed with $\beta$-actin antibody. The graphs indicate the result of densitometric analysis and the data of protein is expressed as the ratio of HIF-1 $\alpha$ (A) and $\mathrm{Cbf} \alpha 1$ (B) expression to the $\beta$-actin expression, respectively. Similar results were obtained for three independent experiments. The data is the mean \pm S.E.M., $n=3$ per group, $* p<0.05, * * p<0.01$ compared with siRNA group, respectively. ${ }^{\#} p<0.01$ compared with the normoxia siRNA group.

centration is an important component of the stem cell niche (Peerani and Zandstra 2010; Santilli et al. 2010). In the present study, hypoxia provokes MSCs proliferation, and increases ALP activity and production of both Col I/III. Both ALP and Col I/III are the hallmarks of MSCs differen- 
tiation into osteogenic lineage cells. Though the differentiation of MSCs has not been directly examined in the present study, the increased production of Col I/III and the increased activity of ALP reflect the properties of the osteogenic cells. Thus, we are supposed that hypoxia enhances MSCs to differentiate into osteogenic lineage cells. Under hypoxia condition, MSCs can be induced into osteogenic lineage cells and provide a potential clinical application to cure bone defects.

Hypoxic signaling is mediated by HIF-1, a heterodimer of HIF- $1 \alpha$ and HIF- $1 \beta$ (Harris 2002). HIF- $1 \alpha$ expression is regulated by oxygen levels, whereas HIF- $1 \beta$ is constitutively expressed. Under normoxia condition, HIF- $1 \alpha$ is degraded promptly through the ubiquitin-proteasome system with low cytoplasm content. When oxygen stress decreases, the degradation of HIF- $1 \alpha$ is inhibited and replaced with HIF-1 $\beta$. As a result, it is activated and translocated into the nucleus and mediates the transcription of numerous hypoxia-response genes, such as $\mathrm{Cbf} \alpha 1$ and vascular endothelial growth factor (VEGF) (Le et al. 2004).

Both HIF-1 $\alpha$ and VEGF are the key components in the process of MSCs-related bone development and regeneration (Wan et al. 2010). VEGF, a transcriptional target of HIF- $1 \alpha$, plays an important role in the angiogenesis. Osteogenesis is tightly coupled with angiogenesis during bone development and regeneration. It has been reported that over-expression of $\mathrm{Cbf} \alpha 1$ in fibroblasts induces VEGF production by stimulating VEGF transcription (Zelzer et al. 2001; Chung et al. 2010). In the present study, we also showed that at the later stage of hypoxia exposure, $\mathrm{Cbf} \alpha 1$ expression was increased, but whether VEGF is involved in the differentiation of MSCs into osteogenic lineages remains to be investigated.

$\mathrm{Cbf} \alpha 1$ is an important protein closely associated with bone development and regeneration, and its expression was significantly increased at the later stage of hypoxia treatment, while HIF- $1 \alpha$ expression began to decrease. In addition, down-regulation of HIF- $1 \alpha$ with SiRNA profoundly induced $\mathrm{Cbf} \alpha 1$ expression either under normoxia or hypoxia condition. These data suggest that $\mathrm{Cbf} \alpha 1$ could induce the differentiation of MSCs into osteogenic lineage cells, and that HIF- $1 \alpha$ negatively regulates $\mathrm{Cbf} \alpha 1$ expression, being consistent with previous reports that hypoxia can decrease Runx $2 / \mathrm{Cbf} \alpha 1$ expression in human osteoblast-like cells (Park et al. 2002). Cbf $\alpha 1$, a target gene of HIF- $1 \alpha$, is a crucial factor in osteogenesis and bone development, and also can enhance the ossification process. However, Pieter et al (2007) and Nekanti et al (2010) have demonstrated that HIF-1 $\alpha$ can improve the differentiation of MSCs into osteogenic lineages (Pieter et al. 2007; Nekanti et al. 2010), which seems to be contrary to our findings. From this point of view, it is thought to be that HIF- $1 \alpha$ negatively regulated the expression of $\mathrm{Cbf} \alpha 1$, and $\mathrm{Cbf} \alpha 1$, rather than HIF- $1 \alpha$, directly enhances the differentiation of MSCs into osteogenic lineages.

In conclusion, in the present study, we have demon- strated that hypoxia induces the differentiation of MSCs into osteogenic lineage cells and increases ALP activity and production of $\mathrm{Col} \mathrm{I} / \mathrm{III}$. We also suggest that $\mathrm{Cbf} \alpha 1$ may mediate the differentiation of MSCs into osteogenic lineage cells.

\section{Acknowledgment}

This work was supported by Natural Science Foundation Plan of Chongqing (CSTC, 2008BB5227).

\section{Conflict of Interest}

All authors have no conflict of interest.

\section{References}

Augello, A. \& De Bari, C. (2010) The regulation of differentiation in mesenchymal stem cells. Hum. Gene Ther, 21, 1226-1238.

Chung, D.J., Hayashi, K., Toupadakis, C.A., Wong, A. \& Yellowley, C.E. (2010) Osteogenic proliferation and differentiation of canine bone marrow and adipose tissue derived mesenchymal stromal cells and the influence of hypoxia. Res. Vet. Sci., (in press).

Granero-Molto, F., Weis, J.A., Longobardi, L. \& Spagnoli, A. (2008) Role of mesenchymal stem cells in regenerative medicine: application to bone and cartilage repair. Expert Opin. Biol. Ther, 8, 255-268.

Haddad, J. \& Harb, H. (2005) Cytokines and the regulation of hypoxia-inducible factor (HIF)-1 $\alpha$. Int. Immunopharmacol., 5, 461-483.

Harris, A.L. (2002) Hypoxia-a key regulatory factor in tumour growth. Nat. Rev. Cancer, 2, 38-47.

Huang, Z., Ren, P.G., Ma, T., Smith, R.L. \& Goodman, S.B. (2010) Modulating osteogenesis of mesenchymal stem cells by modifying growth factor availability. Cytokine, 51, 305-310.

Le, Q.T., Denko, N.C. \& Giaccia, A.J. (2004) Hypoxic gene expression and metastasis. Cancer Metastasis Rev., 23, 293-310.

Lee, K.D., Kuo, T.K., Whang-Peng, J., Chung, Y.F., Lin, C.T., Chou, S.H., Chen, J.R., Chen, Y.P. \& Lee, O.K. (2004) In vitro hepatic differentiation of human mesenchymal stem cells. Hepatology, 40, 1275-1284.

Liu, L., Yu, Q., Lin, J., Lai, X., Cao, W., Du, K., Wang, Y., Wu, K., Hu, Y., Zhang, L., Xiao, H., Duan, Y. \& Huang, H. (2011) HIF- $1 \alpha$ is essential for hypoxia-induced mesenchymal stem cell mobilization into the peripheral blood. Stem. Cells Dev., In press.

Mikami, Y., Ishii, Y., Watanabe, N., Shirakawa, T., Suzuki, S., Irie, S., Isokawa, K. \& Honda, M.J. (2011) CD271/p75(NTR) Inhibits the differentiation of mesenchymal stem cells into osteogenic, adipogenic, chondrogenic, and myogenic lineages. Stem. Cells Dev., [Epub ahead of print].

Mohyeldin, A., Garzón-Muvdi, T. \& Quiñones-Hinojosa, A. (2010) Oxygen in stem cell biology: a critical component of the stem cell niche. Cell Stem. Cell, 7, 150-161.

Nagano, M., Kimura, K., Yamashita, T., Ohneda, K., Nozawa, D., Hamada, H., Yoshikawa, H., Ochiai, N. \& Ohneda, O. (2010) Hypoxia responsive mesenchymal stem cells derived from human umbilical cord blood are effective for bone repair. Stem. Cells Dev., 19, 1195-1210.

Nekanti, U., Dastidar, S., Venugopal, P., Totey, S. \& Ta, M. (2010) Increased proliferation and analysis of differential gene expression in human Wharton's jelly-derived mesenchymal stromal cells under hypoxia. Int. J. Biol. Sci., 6, 499-512.

Otto, W.R. \& Rao, J. (2004) Tomorrow's skeleton staff: mesenchymal stem cells and the repair of bone and cartilage. Cell Prolif., 37, 97-110.

Park, J.H., Park, B.H., Kim, H.K., Park, T.S. \& Baek, H.S. (2002) 
Hypoxia decreases Runx2/Cbf $\alpha 1$ expression in human osteoblast-like cells. Mol. Cell. Endocrinol., 192, 197-203.

Peerani, R. \& Zandstra, P.W. (2010) Enabling stem cell therapies through synthetic stem cell-niche engineering. J. Clin. Invest., 120, 60-70.

Pieter, J., Emansa, F.S., Don, A.M., Keryn, M.R., Andy, C., Lodewijk, W.R., Sjoerd, K.B., Willem, V. \& Roel, K. (2007) A novel in vivo model to study endochondral bone formation; HIF- $1 \alpha$ activation and BMP expression. Bone, 40, 409-418.

Potier, E., Ferreira, E., Andriamanalijaona, R., Pujol, J.P., Oudina, K., Logeart-Avramoglou, D. \& Petite, H. (2007) Hypoxia affects mesenchymal stromal cell osteogenic differentiation and angiogenic factor expression. Bone, 40, 1078-1087.

Qi, M.C., Zou, S.J., Han, L.C., Zhou, H.X. \& Hu, J. (2009) Expression of bone-related genes in bone marrow MSCs after cyclic mechanical strain: implications for distraction osteogenesis. Int. J. Oral Sci., 1, 143-150.

Rabie, A.B., Tang, G.H. \& Hägg, U. (2004) Cbfa1 couples chondrocytes maturation and endochondral ossification in rat mandibular condylar cartilage. Arch. Oral Biol., 49, 109-118.
Santilli, G., Lamorte, G., Carlessi, L., Ferrari, D., Rota, N.L., Binda, E., Delia, D., Vescovi, A.L. \& Filippis, L. (2010) Mild hypoxia enhances proliferation and multipotency of human neural stem cells. PLoS One, 5, e8575.

Seong, J.M., Kim, B.C., Park, J.H., Kwon, I.K., Mantalaris, A. \& Hwang, Y.S. (2010) Stem cells in bone tissue engineering. Biomed. Mater., 5, 062001.

Wan, C., Shao, J., Gilbert, S.R., Riddle, R.C., Long, F., Johnson, R.S., Schipani, E. \& Clemens, T.L. (2010) Role of HIF-1 $\alpha$ in skeletal development. Ann. N.Y. Acad. Sci., 1192, 322-326.

Zelzer, E., Glotzer, D.J., Hartmann, C., Thomas, D., Fukai, N., Soker, S. \& Olsen, B.R. (2001) Tissue specific regulation of VEGF expression during bone development requires $\mathrm{Cbf} \alpha 1 /$ Runx2. Mech. Dev., 106, 97-106.

Zhao, Z.G., Zhao, S.M., Xu, N., Liu, X.Y., Liao, W.Q. \& Jia, W. (2010) Lovastatin improves neurological outcome, reduces neurodegeneration, enhances M/NMDA receptor binding activity after nucleus basalis magnocellularis lesion in rats. Neuroscience, 167, 954-963. 